\title{
Avaliação formativa sob a ótica do tutor
}

\section{Formative evaluation under the optics of the tutor}

\author{
Marcia Bitar Portella', Lidiane da Silva Sabathe', Jade Beatriz Iwasaka-Neder', \\ Pedro Luis Iwasaka-Neder' \\ 'Universidade do Estado do Pará, Belém (PA), Brasil.
}

\section{RESUMO}

Objetivo: Analisar o processo de avaliação formativa do estudante de medicina, realizado pelo docente, nas sessões tutoriais, em duas Instituições de Ensino Superior. Métodos: Pesquisa quantitativa, analítica e transversal. Trinta e cinco tutores participaram da pesquisa realizada em duas Instituições de Ensino Superior. A coleta de dados foi realizada por meio da aplicação de questionário com questões objetivas. Resultados: A maioria dos tutores acredita no potencial da avaliação formativa. Julga alcançar seus objetivos (identificar as dificuldades - 91,4\%; apresentar as dificuldades - 77,1\%; sugerir estratégias para superação de dificuldades - 71,4\%; avaliar de forma contínua e progressiva - 94,2\%), contribuindo com o desenvolvimento do aluno (88,5\%) e procura manter-se atualizada, participando de capacitações $(97,1 \%)$ e trocando experiências com os colegas $(97,1 \%)$. Consideram-se capacitados (60\%) ou apenas parcialmente capacitados (40\%), tendo dúvidas quanto a sua subjetividade $(62,8 \%)$, imparcialidade $(42,8 \%)$, coerência $(40 \%)$ e quanto à efetividade do processo (22,8\%). Muitos tutores $(54,2 \%)$ acreditam que os alunos não compreendem/aceitam totalmente a avaliação formativa. Conclusão: A maioria dos tutores acredita no potencial da avaliação formativa, porém, ainda há muitos desafios a serem superados, o que os leva a nem sempre sentirem-se seguros ao dar o feedback. Esta pesquisa contribui com a superação destes desafios, ao possibilitar uma maior compreensão dos fatores dificultadores e apontar novos rumos a serem seguidos. Pesquisas que avaliem as dificuldades dos alunos neste processo são necessárias à definição de estratégias mais abrangentes

Recebido: Ago. 13, 2017 Aceito: Nov. 20, 2017

\section{COMO CITAR ESTE ARTIGO}

Portella MB, Sabathe LS, Iwasaka-Neder JB, Iwasaka-Neder PL. Avaliação formativa sob a ótica do tutor. Interdisciplinary Journal of Health Education. 2017 Jan-Jul;2(1):45-53. http:// dx.doi.org/10.4322/ijhe.2016.028

\section{CORRESPONDÊNCIA}

Marcia Bitar Portella

Faculdade Metropolitana da Amazônia Av. Visc. de Souza Franco, 72, Reduto, CEP 66053-000, Belém (PA), Brasil marciabitar@gmail.com

\section{FONTE DE FINANCIAMENTO Bolsa PROPESP/UEPA para} Lidiane da Silva Sabathe.

\section{CONFLITO DE INTERESSE}

Os autores declararam não haver conflitos de interesse.

O estudo foi realizado na Universidade do Estado do Pará e na Faculdade Metropolitana da Amazônia, Belém (PA), Brasil.

Este trabalho é resultado de dissertação de Mestrado de Ensino em Saúde de Lidiane da Silva Sabathe, sob orientação de Dra. Marcia Bitar Portella.
PALAVRAS-ChaVe: Educação médica. Aprendizagem baseada em problemas. Feedback formativo. Tutoria.

\section{ABSTRACT}

Objective: To analyze the process of formative evaluation of the medicine student, conducted by the teacher, in the tutorial sessions, in two Institutions of Higher Education. Methods: Quantitative, analytical and cross-sectional research. Thirty-five tutors participated in the research conducted in two Higher Education Institutions. The data collection was performed through the application of a questionnaire with objective questions. Results: It was observed that the majority believes in the potential of formative evaluation. (to identify difficulties $-91.4 \%$, to present the difficulties $-77.1 \%$, to suggest strategies for overcoming difficulties $-71.4 \%$, to evaluate continually and progressively $-94.2 \%)$, contributing to student development (88.5\%) and seeking to keep updated by participating in trainings $(97.1 \%)$ and exchanging experiences with colleagues (97.1\%). They are considered to be trained (60\%) or only partially qualified (40\%), having doubts about their subjectivity (62.8\%), impartiality (42.8\%), coherence (40\%) and the effectiveness of the process $(22.8 \%)$. Most of the teachers (54.2\%) believe that the students do not fully understand / accept the formative evaluation. Conclusion: Most tutors believe in the potential of formative assessment, however, there are still many challenges to be overcome, which leads them to not always feel secure in giving feedback. This research deals with overcoming these challenges, by allowing a greater understanding of the factors that make it difficult and pointing out new directions to be followed. Research that evaluates students' difficulties in this process is necessary to define more comprehensive strategies.

KEYWORDS: Medical education. Problem-based learning. Formative feedback. Mentoring. 


\section{Introdução}

O processo de avaliação é considerado uma das dimensões estratégicas para a mudança na educação médica, juntamente com a docência, a gestão acadêmica e o processo de ensino-aprendizagem ${ }^{1}$. Não se pode realizar maiores reformas pedagógicas sem levar em conta os sistemas e as práticas de avaliação, sem integrá-los à reflexão e modifica-los simultaneamente ${ }^{2}$.

Mudanças vêm sendo implantadas visando nos auxiliar na árdua tarefa de formar médicos aptos a atender aos desafios da sociedade atual, que a cada dia exige mais da classe médica ${ }^{3}$. Elas devem ser feitas levando-se em consideração as especificidades dos estudantes da geração atual ${ }^{3}$, a chamada "Geração $Y$ ", composta por jovens que, por já terem nascido imersos em tecnologia, viverem conectados e usufruírem de acesso instantâneo à informação, são mais críticos e questionadores, e exigem relacionamentos francos e diretos ${ }^{4}$.

O processo de avaliação deve ser abrangente e variado o suficiente para contemplar as diversas competências esperadas do médico, que são amplo conhecimento científico; habilidade para cuidar do paciente com compaixão e afetividade; busca de melhora contínua em seu atendimento, a partir da autoavaliação constante e busca do aprendizado ao longo de toda a vida; habilidades de comunicação com paciente, familiares e outros profissionais; profissionalismo, demonstrado pela integridade e respeito ao próximo e capacidade de utilizar, de maneira eficaz, os recursos do sistema de saúde ${ }^{5,6}$.

Após extensas discussões com variados setores da sociedade, as Diretrizes Curriculares Nacionais para o Curso de Medicina (DCNCM) vieram nortear as mudanças necessárias e estabelecem que o processo de avaliação deva ter como objetivo construir e ressignificar saberes, requerendo "maior horizontalização, ação cooperativa, solidária e ética, postura ativa, crítica e reflexiva, além do desenvolvimento da capacidade de aprender a aprender, de identificar os próprios valores e de abrir-se para a superação de limites e constrições" ${ }^{\prime 7}$.

Neste contexto, a avaliação formativa - que se caracteriza pelo acompanhamento contínuo do aluno, permitindo a identificação de dificuldades e a busca de soluções ao longo de todo o percurso, e não apenas ao seu final, estimulando a atuação do aluno como sujeito de sua avaliação e tornando-o consciente do desenvolvimento de sua aprendizagem ${ }^{8}$ - tem sido considerada uma fundamental complementação à avaliação somativa9.

No entanto, apesar de todo este potencial, seu estabelecimento não tem ocorrido com a fluidez e celeridade desejadas. Docentes, discentes e até mesmo instituições ainda demostram desconforto e insegurança com sua prática. A capacitação ausente ou insipiente, por um lado, e a resistência em aceitar novas práticas, por outro, cria intensos desafios?.

Os enormes benefícios propiciados pela avaliação formativa, bem como as dificuldades em sua realização, despertaram o interesse para estudo deste tema, pois uma maior compreensão de seus fatores dificultadores pode contribuir com a mudança deste cenário, ao apontar novos rumos a serem seguidos, permitindo que docentes e discentes possam aproveitar ao máximo todo o potencial desta metodologia, que permite avaliações de qualidade, oportunas e orientadoras, sendo auxiliares legítimas da construção do conhecimento em aspecto amplo - não apenas dos conteúdos propriamente ditos, mas também, de posturas e atitudes.

Portanto, este estudo tem como objetivo analisar o processo de avaliação formativa do estudante de medicina, realizado pelo docente, nas sessões tutoriais, em duas Instituições de Ensino Superior (IES); conhecer as concepções dos tutores sobre avaliação formativa e investigar as possíveis dificuldades técnicas do professor no processo de avaliação formativa na sessão tutorial. 


\section{Metodologia}

Trata-se de uma pesquisa quantitativa, realizada por meio de um estudo analítico e transversal, realizada em duas IES, localizadas na cidade de Belém, no estado do Pará, sendo uma pública (IES 1) e uma privada sem fins lucrativos (IES 2). Os participantes da pesquisa foram os professores que atuavam nas sessões tutoriais dos dois referidos cursos. A seleção da amostra foi feita por convite direto da pesquisadora aos professores, nas IES. Todos os tutores que desejassem participar poderiam ser incluídos na pesquisa (amostra aleatória simples). De um universo de 48 tutores, 35 aceitaram participar da pesquisa. Critérios de inclusão: Exercer a função de tutor nas sessões tutoriais e aceitar participar da pesquisa por meio da assinatura do Termo de Consentimento Livre e Esclarecido (TCLE). A pesquisa foi autorizada pelos coordenadores de cursos das duas IES e aprovada por Comitê de Ética em Pesquisa, obedecendo à resolução número 466/12 do Conselho Nacional de Saúde pelo número CAAE: 66959517.0.0000.5701. A coleta de dados foi realizada por meio da aplicação de questionário com questões objetivas, onde o entrevistado tinha a possibilidade de justificar sua resposta, e ocorreu em maio de 2017. Os questionários foram entregues aos tutores nas salas dos professores ou nas salas de tutorias e, posteriormente, foram devolvidos aos pesquisadores nestes mesmos locais, ou entregues na sala da coordenação dos cursos. Três questionários foram enviados e recebidos via e-mail. Foi realizado um estudo piloto com seis tutores, sendo detectada a necessidade de realizar mudanças na redação de algumas perguntas, e adequou-se o questionário, de forma que pudesse responder aos objetivos da pesquisa. A análise estatística foi realizada através do teste Qui-Quadrado ou $G$ (aderência), sendo que resultados com $\mathrm{p} \leq 0,05$ foram considerados estatisticamente significativos.

\section{Resultados}

Foram entrevistados 35 tutores das duas IES. Destes, 34 (97,14\%; $\mathrm{p}<0,05)$ afirmaram ter participado de capacitação em tutoria, porém, somente $21(60 \%)$ consideram-se capacitados e $14(40 \%)$ consideram-se apenas parcialmente capacitados a realizar a avaliação formativa $(\mathrm{p}>0,05)$.

A maioria dos docentes tem o hábito de trocar informações sobre avaliação formativa com outros tutores $(97,1 \%$; $<<0,05)$, considera-se comprometida com o processo de avaliação $(82,8 \% ; \mathrm{p}<0,05)$ e acredita estar contribuindo com o desenvolvimento do aluno $(88,5 \% ; \mathrm{p}<0,05)$.

Muitos julgam alcançar os objetivos da avaliação formativa - identificar $(91,4 \% ; p<0,05)$ e apresentar $(77,1 \% ; p<0,05)$ as dificuldades dos alunos, sugerir estratégias para sua superação $(71,4 \% ; \mathrm{p}<0,05)$ e avaliar de forma contínua e progressiva $(94,2 \% ; \mathrm{p}<0,05)$, dados apresentados na Tabela 1.

Tabela 1. Concepções dos tutores quanto ao alcance dos objetivos da avaliação formativa. Belém/PA, 2017.

\begin{tabular}{|c|c|c|c|c|c|c|c|c|}
\hline \multirow{2}{*}{$\begin{array}{l}\text { Você consegue alcançar } \\
\text { os objetivos da avaliação } \\
\text { formativa, em sua tutoria? }\end{array}$} & \multicolumn{2}{|c|}{ a } & \multicolumn{2}{|c|}{ b } & \multicolumn{2}{|c|}{ c } & \multicolumn{2}{|c|}{ d } \\
\hline & $\mathbf{n}$ & $\%$ & $\mathbf{n}$ & $\%$ & n & $\%$ & $\mathbf{n}$ & $\%$ \\
\hline Sim & 32 & 91,43 & 27 & 77,14 & 25 & 71,43 & 33 & 94,29 \\
\hline Não & 0 & 0,00 & 2 & 5,71 & 2 & 5,71 & 1 & 2,86 \\
\hline Parcialmente & 3 & 8,57 & 6 & 17,14 & 8 & 22,86 & 1 & 2,86 \\
\hline P-Valor & \multicolumn{2}{|c|}{$0,0000^{*}$} & \multicolumn{2}{|c|}{$0,0000^{*}$} & \multicolumn{2}{|c|}{$0,0000^{*}$} & \multicolumn{2}{|c|}{$0,0000^{*}$} \\
\hline
\end{tabular}

Item (a) Identifica as dificuldades do aluno, de forma individualizada; Item (b) Apresenta ao aluno suas dificuldades, de forma individualizada; Item (c) Sugere ao aluno, estratégias para superação de suas dificuldades, de forma individualizada; Item (d) Avalia o aluno de forma contínua e progressiva, pontuando sua evolução; ${ }^{*} \mathrm{p}<0,05$. Fonte: Dados resultantes da pesquisa (2017). 
Tabela 2. Concepções dos tutores quanto aos desafios pessoais na avaliação formativa. Belém/PA, 2017.

\begin{tabular}{|c|c|c|c|c|c|c|c|c|c|c|}
\hline \multirow{2}{*}{$\begin{array}{c}\text { Você considera desafios } \\
\text { pessoais, na avaliação } \\
\text { formativa? }\end{array}$} & \multicolumn{2}{|c|}{ a } & \multicolumn{2}{|c|}{ b } & \multicolumn{2}{|c|}{ c } & \multicolumn{2}{|c|}{ d } & \multicolumn{2}{|c|}{ e } \\
\hline & $\mathbf{n}$ & $\%$ & $\mathbf{n}$ & $\%$ & $\mathbf{n}$ & $\%$ & $\mathbf{n}$ & $\%$ & $\mathbf{n}$ & $\%$ \\
\hline Sim & 22 & 62,86 & 15 & 42,86 & 14 & 40,00 & 13 & 37,14 & 8 & 22,86 \\
\hline Não & 4 & 11,43 & 11 & 31,43 & 13 & 37,14 & 12 & 34,29 & 15 & 42,86 \\
\hline Parcialmente & 8 & 22,86 & 8 & 22,86 & 7 & 20,00 & 9 & 25,71 & 12 & 34,29 \\
\hline Não Informado & 1 & 2,86 & 1 & 2,86 & 1 & 2,86 & 1 & 2,86 & 0 & 0,00 \\
\hline P-Valor & \multicolumn{2}{|c|}{$0,0000^{*}$} & \multicolumn{2}{|c|}{$0,0070^{*}$} & \multicolumn{2}{|c|}{$0,0060^{*}$} & \multicolumn{2}{|c|}{0,0170} & \multicolumn{2}{|c|}{0,3470} \\
\hline
\end{tabular}

Item (a) A subjetividade do processo; Item (b) Manter a imparcialidade; Item (c) Ser coerente; Item (d) Ter segurança e tranquilidade ao realizar a devolutiva; Item (e) Suas dúvidas quanto à efetividade do processo; ${ }^{*} \mathrm{p}<0,05$. Fonte: Dados resultantes da pesquisa (2017).

Tabela 3. Concepções dos tutores quanto à compreensão/aceitação, pelo aluno, da avaliação formativa. Belém/PA, 2017.

\begin{tabular}{cccc}
$\begin{array}{c}\text { Na sua visão, o aluno compreende/aceita a } \\
\text { avaliação formativa? }\end{array}$ & $\mathbf{n}$ & $\%$ & P-Valor \\
\hline Sim & 14 & 40,00 \\
\hline Não & 2 & 5,71 & $0,0010^{*}$ \\
\hline Parcialmente & 19 & 54,29 & \\
\hline
\end{tabular}

${ }^{*} \mathrm{p}<0,05$. Fonte: Dados resultantes da pesquisa (2017).

Desafios pessoais são frequentes (Tabela 2), havendo dúvidas quanto a sua subjetividade $(62,8 \%$; $\mathrm{p}<0,05)$, imparcialidade $(42,8 \%$; $<<0,05)$, coerência $(40 \%$; $p<0,05)$ e quanto à efetividade do processo $(22,8 \% ; p>0,05)$. A Tabela 3 mostra que também é significativo o percentual de docentes que acredita que os alunos não compreendem/aceitam totalmente a avaliação formativa $(54,2 \% ; p<0,05)$.

O tempo que destina à avaliação formativa foi considerado adequado por $74,29 \%(p<0,05)$ dos tutores.

\section{Discussão}

Uma avaliação bem feita, com a oferta de um feedback bem realizado, tem enorme impacto no desenvolvimento do aluno ${ }^{10}$, contribuindo com a aquisição e melhoria de competências ${ }^{11}$. Porém, a avaliação formativa consiste em processo complexo, que é parte de uma interação social influenciada por cultura, valores, expectativas, histórias pessoais, grau de relacionamento e poder, sendo considerada um grande desafio ${ }^{12}$. Por isso, apesar de $97 \%$ dos entrevistados afirmarem ter participado de alguma capacitação em tutoria, $40 \%$ dos tutores sentem-se apenas parcialmente capacitados para realizar a avaliação formativa.

Lefroy et al. ${ }^{12}$ concordam que fornecer um bom feebdack não é tarefa fácil, pois ele pode ser visto como uma oportunidade, mas também como uma ameaça, tanto por alunos como por professores. Johnson et al. ${ }^{13}$, ao revisarem a literatura, também identificaram desconforto durante o processo avaliativo, quando professores referiram medo de afetar a autoestima do aluno e desencadear uma resposta defensiva, prejudicando a relação. Isto os leva, muitas vezes, a considerarem-se malvados.

A falta de capacitação docente é um dos maiores dificultadores da implantação de metodologias ativas de ensino, como a avaliação formativa, pois muitas escolas médicas realizaram suas reformas curriculares com pouco investimento neste aspecto ${ }^{14-16}$. Além da participação em cursos, o tutor também pode manter-se capacitado e melhorar 
suas habilidades de dar feedback através da reflexão, ao final de cada sessão, sobre seu desempenho na avaliação, questionando-se sobre o que fez bem, o que pode melhorar e quais estratégias podem ser utilizadas nas próximas sessões ${ }^{17}$.

A troca de experiência com outros tutores, que costuma ser realizada por quase todos os entrevistados $(97,14 \%)$, enriquece esta reflexão, bem como estar aberto para receber feedback de seus alunos, colegas e superiores. Uma interessante forma de realizar esta troca de experiências é participar, como observador, do momento da avaliação realizada por outro colega, para posterior discussão. Leitura de artigos sobre o tema também é uma estratégia útil ${ }^{17}$.

Outro fator que influencia a qualidade da avaliação formativa é o compromisso que o tutor assume com o processo. Apesar de a maioria dos entrevistados considerar-se comprometida, $17,14 \%$ afirmam comprometer-se apenas parcialmente. Fonseca et al. ${ }^{18}$ destaca que, apesar do enorme potencial deste método, muitos professores não costumam desenvolvê-lo de maneira adequada.

Embora quase metade dos tutores não se considere totalmente capacitados, $88,57 \%$ creem na significativa contribuição de sua avaliação para o desenvolvimento de seu aluno e, a maioria, acredita alcançar os principais objetivos desta. Narciss ${ }^{11}$ identificou como os três principais aspectos que influenciam no resultado do feedback, o ambiente instrucional (definição clara dos objetivos, conteúdos e tarefas de aprendizagem, com padrões de avaliação bem estabelecidos); a estratégia utilizada pelo tutor (o âmbito e a natureza das informações, a forma de apresentação); e as particularidades do aluno (conhecimento prévio, nível de competência, de metacognição, de motivação).

O primeiro passo para a realização de uma avaliação satisfatória, com adequada identificação das dificuldades dos alunos, é a definição exata do que se deseja avaliar, quais os conteúdos e competências, com o estabelecimento claro de objetivos e metas, bem como dos padrões desejados ${ }^{16}$, que deverão ser mensurados através de estratégias e instrumentos válidos e confiáveis ${ }^{11}$.

Considerando-se as estratégias utilizadas pelo tutor outro ponto fundamental, Johnson et al. ${ }^{13}$ buscaram identificar alguns comportamentos que podem diminuir o potencial da avaliação. Destacam a realização de observações não específicas, com comentários do tipo "você foi muito bem" ou "você precisa melhorar um pouco", sem apontar o que foi feito corretamente e o que precisa ser melhorado; a não elaboração de plano de melhoria; a não estimulação da participação ativa do aluno, ao fazer observações e recomendações através de monólogos, dando pouco espaço para falas e perguntas do aluno, não valorizando a autoavaliação e não colaborando para que participem ativamente das decisões. Então, o tutor deve buscar promover um ambiente de confiança e respeito, procurando compreender o que o aluno espera do ensino e trabalhando em conjunto, através de um diálogo maduro, no estabelecimento de regras básicas, objetivos e metas, estimulando-os a atingirem os resultados de forma independente ${ }^{16}$.

Assim como relatado por muitos de nossos docentes, expor as dificuldades dos alunos também foi sentido como um desafio pelos entrevistados por Qadan et al. ${ }^{19}$, o que os leva, muitas vezes, a não serem sinceros em suas avaliações. Os autores acreditam que isto pode estar relacionado com a falta de cultura, no meio médico, de dar feedback; à pressão por se dizer apenas o que o outro quer ouvir; ao desconforto em lidar com a insatisfação do estudante e ao receio de receber uma avaliação negativa do aluno, o que poderia interferir com suas possibilidades de promoção institucional. E alertam para os riscos de esta prática resultar em falsas garantias do desenvolvimento de competências, impedindo que o aluno atinja seu pleno potencial.

Desta forma, sugere-se que, ao expor as dificuldades dos alunos, o tutor parta dos aspectos onde houve bom desempenho, seguidos por aqueles que precisam ser melhorados. Destacar os aspectos positivos com maior frequência que os negativos geram um ambiente emocional favorável a melhores resultados ${ }^{20}$. 
Por este ser um desafio amplamente relatado na literatura, vários modelos de feedback tem sido publicados, visando diminuir a resposta defensiva e preservar a autoestima e motivação do aluno.

Um modelo simples é o Feedback Sanduíche, que consiste em iniciar com um comentário positivo, em seguida, fazer uma crítica e, por fim, fazer novo comentário positivo ${ }^{21}$. Tem a intenção de suavizar a mensagem que pode ser vista como negativa, porém, alguns autores relatam o risco de os alunos não darem atenção ao feedback corretivo e alcançar menor resultado no desempenho ${ }^{22}$.

Outra estratégia recomendada é iniciar solicitando que o aluno faça sua autoavaliação. Neste momento, o tutor pode guiá-lo através de perguntas reflexivas, como sobre o que ele percebe que fez bem e quais aspectos podem ser melhorados. O aluno, frequentemente, destaca os fatos que o professor pretendia discutir, tornando mais fácil a sua abordagem ${ }^{16}$.

French et al. ${ }^{23}$ sugere estratégia semelhante, chamada de Ask-Tell-Ask, que é composta por três passos. No primeiro passo, o professor pergunta ao aluno sobre sua autoavaliação. No passo seguinte, o professor comenta as observações do aluno, faz suas próprias observações, sempre destacando pelo menos uma coisa que o aluno fez bem, e pontuando as áreas que precisam ser melhoradas (no máximo uma a duas). Por fim, no último passo, checa se o aluno compreendeu o que foi conversado e discute um plano de melhora. $\mathrm{O}$ autor percebe que este modelo desenvolve a reflexão, a responsabilidade, as habilidades de monitorização de performance e de autoavaliação.

A análise deve ser focada no desempenho da tarefa - evitando-se referências a características pessoais - informando com clareza as discrepâncias em relação aos objetivos a serem alcançados. É fundamental usar um tom respeitoso, de suporte, além de buscar compreender as possíveis razões para estas dificuldades ${ }^{13,23}$.

É importante que se procure conhecer o aluno, sua personalidade e seu temperamento ${ }^{16}$ e que se esteja atento às reações emocionais despertadas pelo feedback. Estas reações devem ser identificadas e discutidas, de forma respeitosa e empática, sendo esperado que o tutor tenha alguma habilidade no gerenciamento de conflitos ${ }^{24}$.

A exposição das dificuldades constatadas sempre deve vir acompanhada de sugestões de como superá-las ${ }^{25}$, que devem ser intencionais, significativas e compatíveis com o grau de compreensão dos alunos ${ }^{23}$. A elaboração do plano de ação deve ser realizada em conjunto com o aluno, e vai depender de seu grau de desenvolvimento. Para alunos mais desenvolvidos, devem-se fazer questionamentos que os auxiliem a identificar os passos a serem dados. Já para alunos menos desenvolvidos, é provável que seja necessário fornecer orientações específicas ${ }^{12}$. As medidas definidas pelo aluno serão reforçadas ou modificadas pelo professor, de acordo com a necessidade, até que, juntos, possam elaborar um plano de ação para atingir os resultados desejados ${ }^{13}$.

A maioria dos tutores refere avaliar o aluno de forma contínua e progressiva, pontuando a sua evolução $(94,29 \%)$. Este acompanhamento ao longo do tempo é fundamental e deve auxiliar o discente a responder, constantemente, a três questões principais: Para onde eu devo ir (quais os objetivos)? Como estou indo (quais os progressos em relação aos objetivos)? E qual o próximo passo (quais atividades precisam ser realizadas para um melhor desempenho)? ${ }^{23}$

Nesta avaliação contínua, o tutor deve verificar o grau de motivação do aluno em colocar em prática o plano de ação elaborado, checando se ele concorda com a necessidade de mudar, se deseja fazer esta mudança e se julga estar apto a realizá-la ${ }^{12}$.

Os frequentes desafios relatados pelos entrevistados são justificados, pois estudos mostram que os discentes costumam ser críticos em relação às orientações fornecidas, analisando o grau de comprometimento do tutor, avaliando se há real interesse pelo seu crescimento, ao dar o feedback. Consideram não apenas as características profissionais, mas também, pessoais, desejando confiança, respeito e carinho, valorizando a empatia, a disposição a ouvir e a valorizar as ideias dos outros, sendo 
estes elementos fundamentais para a aceitação de sugestões que despertam maior resistência ${ }^{26}$. Então, para que haja segurança e tranquilidade no momento da avaliação, o tutor deve investir na relação professor-aluno ${ }^{27}$. Além disso, é fundamental ter domínio do conteúdo a ser estudado, bem como estar capacitado a utilizar técnicas pedagógicas adequadas à aprendizagem dos estudantes ${ }^{15}$.

Visando aumentar a confiabilidade, muitas instituições, inclusive as estudadas, vêm utilizando a avaliação realizada por múltiplos observadores - avaliação pelo tutor, avaliação interpares e autoavaliação. A autoavaliação e a avaliação interpares capacitam o aluno a julgar seu próprio trabalho, bem como o trabalho de outros ${ }^{3}$.

Assim como muitos tutores tem dúvidas quanto à efetividade da avaliação formativa, a maioria acredita que os alunos a compreendem/aceitam apenas parcialmente $(54,2 \%)$ ou não a compreendem/aceitam (5,7\%). De fato, Baik et al. ${ }^{28}$ constataram que quase metade dos estudantes universitários da Austrália estão menos satisfeitos com a avaliação e o feedback de que com qualquer outro aspecto de seus cursos. Porém, esta falta de aceitação não ocorre porque os alunos não a consideram importante, mas porque sua prática ainda não tem a qualidade desejada ${ }^{25}$. Outro aspecto que contribui para sua pouca aceitação é a influência negativa exercida pela cultura da avaliação somativa, onde o principal objetivo é ser aprovado, mais do que aprender, o que desperta o medo do fracasso e da punição potencial, e os leva a evitar o feedback ${ }^{28}$.

Quando os alunos não desenvolvem a compreensão necessária, eles não dão credibilidade às orientações e não seguem os conselhos, seja por não acreditarem na necessidade de mudar ou mesmo por não saberem como fazê-lo, podendo ocorrer, até mesmo, piora no desempenho. Sentimentos de raiva, ansiedade, vergonha, frustração e desmotivação são frequentemente relatados ${ }^{13}$. As instituições devem implementar um debate contínuo sobre a qualidade e o valor do feedback, levando-se em consideração, não apenas a forma como ele é fornecido pelo tutor, mas também, sobre como o aluno tem sido preparado e capacitado para recebê-lo ${ }^{29}$. O discente deve ser conscientizado de seu papel central no processo. Deve ser estimulado a atuar de forma ativa, indo em busca do feedback, e não apenas esperando por ele. Ao receber orientações, precisa solicitar maiores esclarecimentos, sempre que achar necessário, e analisá-los de acordo com suas próprias observações em relação ao seu desempenho. Pode, inclusive, buscar a opinião de terceiros, antes de definir quais aspectos são realmente úteis ao seu crescimento. Deve saber diferenciar um "bom", de um "pobre" feedback ${ }^{29}$.

A qualidade do instrumento de avaliação é essencial para a mensuração de competências de forma válida e confiáve ${ }^{11}$. A maioria dos docentes deseja trabalhar com um formulário de avaliação, porém, estes dificilmente atendem suas necessidades. Quando são obrigados a trabalhar com regras com as quais não concordam, eles não as respeitam, ou não conseguem estabelecer padrões claros em sua avaliação $0^{30}$.

A maioria dos tutores afirma dedicar tempo suficiente à avaliação formativa, porém, $25 \%$ não estão satisfeitos com este aspecto. É importante que as observações sejam realizadas logo após o evento, podendo haver um horário reservado especificamente para sua realização ${ }^{29}$, além de serem realizadas em outros momentos em que houver necessidade. $\mathrm{O}$ tempo insuficiente pode favorecer a um feedback muito direto, dado pelo professor, sem que haja espaço para a troca desejada.

\section{Conclusão}

A maioria dos tutores acredita no potencial da avaliação formativa, porém, ainda há muitos desafios a serem superados, o que os leva a nem sempre sentirem-se seguros ao dar o feedback. Esta pesquisa contribui com a superação destes desafios, ao possibilitar uma maior compreensão dos fatores dificultadores e apontar novos rumos a serem seguidos. Pesquisas que avaliem as dificuldades dos alunos neste processo são necessárias à elaboração de estratégias mais abrangentes. 


\section{Referências}

1. Aguiar AC. Cultura de avaliação e transformação da educação médica: a ABEM na interlocução entre academia e governo. Rev Bras Educ Med. 2006;30(2):98-101. http://dx.doi.org/10.1590/S0100-55022006000200013.

2. Perrenoud P. Avaliação: da excelência à regulação das aprendizagens: entre duas lógicas. Porto Alegre: Artes Médicas; 1999.

3. Ferris HA, O’Flynn D. Assessment in medical education: what are we trying to achieve? High Educ [Internet]. 2015;4(2):13944 [citado em 2017 Ago 13]. Disponível em: http://www.sciedu.ca/journal/index.php/ijhe/article/view/6662/0

4. Teixeira CH. Os desafios da educação para as novas gerações: entendendo a geração Y. Qualis Sumaré. 2016;5(1):1-5.

5. Norcini J, Anderson B, Bollela V, et al. Criteria for good assessment: consensus statement and recommendations from the Ottawa 2010 Conference. Med Teach. 2011;33(3):206-14. http://dx.doi.org/10.3109/0142159X.2011.551559. PMid:21345060.

6. Gontijo ED, Alvim C, Megale L, Melo JRC, Lima MECC. Matriz de competências essenciais para a formação e avaliação de desempenho de estudantes de medicina. Rev Bras Educ Med. 2013;37(4):526-39. http://dx.doi.org/10.1590/ S0100-55022013000400008.

7. Ministério da Educação. Conselho Nacional de Educação. Diretrizes curriculares nacionais do curso de graduação em medicina [Internet]. Brasília; 2014 [citado em 2017 Ago 13]. Disponível em: http://portal.mec.gov.br/conselho-nacional-de-educacao/ atos-normativos--sumulas-pareceres-e-resolucoes?id=20138

8. Cappelletti IF. Opções metodológicas em avaliação: saliências e relevâncias no processo decisório. Roteiro. 2012;37(2):211-26.

9. Borges MC, Miranda CH, Santana RC, Bollela VR. Avaliação formativa e feedback como ferramenta de aprendizado na formação de profíssionais da saúde. Medicina. 2014;47(3):324-31.

10. Evans C. Making sense of assessment feedback in higher education. Rev Educ Res. 2013;83(1):70-120. http://dx.doi. org/10.3102/0034654312474350.

11. Narciss S. Conditions and effects of feedback viewed through the lens of the interactive tutoring feedback model. In: Carless D, Bridges S, Chan CKY, Glofcheski R, editores. Scaling up assessment for learning in higher education. Dordrecht: Springer; 2016. p. 173-89.

12. Lefroy J, Watling C, Teunissen PW, Brand P. Guidelines: the do's, don'ts and don't knows of feedback for clinical education. Perspect Med Educ. 2015;4(6):284-99. http://dx.doi.org/10.1007/s40037-015-0231-7. PMid:26621488.

13. Johnson CE, Keating JL, Boud DJ, et al. Identifying educator behaviours for high quality verbal feedback in health professions education: literature review and expert refinement. BMC Med Educ. 2016;16(1):96. http://dx.doi.org/10.1186/s12909-0160613-5. PMid:27000623.

14. Perim GL, Abdalla IG, Aguilar-da-Silva RH, Lampert JB, Stella RCR, Costa NMSC. Desenvolvimento docente e a formação de médicos. Rev Bras Educ Med. 2009;33(1, Supl):70-82. http://dx.doi.org/10.1590/S0100-55022009000500008.

15. Vieira JE, Tamousauskas MRG. Avaliação das resistências de docentes a propostas de renovações em currículos de graduação em medicina. Rev Bras Educ Med. 2013;37(1):32-8. http://dx.doi.org/10.1590/S0100-55022013000100005.

16. Hattie J, Timperley H. The power of feedback. Rev Educ Res. 2007;77(1):81-112. http://dx.doi.org/10.3102/003465430298487.

17. Ramani S, Krackov S. Twelve tips for giving feedback effectively in the clinical environment. Med Teach. 2012;34(10):78791. http://dx.doi.org/10.3109/0142159X.2012.684916. PMid:22730899.

18. Fonseca J, Carvalho C, Conboy J, Valente MO, Gama AP. Changing teachers' feedback practices: a workshop challenge. Aust. J. Teach. Educ [Internet]. 2015;40(8):59-82 [citado em 2017 Ago 13]. Disponível em: http://ro.ecu.edu.au/cgi/viewcontent. cgi?article $=2600 \&$ context $=$ ajte

19. Qadan L, Al-Ozairi E, Ayed A, Huang G. Avoiding honest feedback: discordance between formal evaluations and candid assessments of Kuwaiti PBL students. Med Teach. 2013;35(6):459-64. http://dx.doi.org/10.3109/0142159X.2013.774337. PMid:23477472.

20. Voerman L, Meijer PC, Korthagen F, Simons RJ. Promoting effective teacher-feedback: from theory to practice through a multiple component trajectory for professional development. Teach Teach. 2015;21(8):990-1009. http://dx.doi.org/10.108 0/13540602.2015.1005868.

21. Docheff DM. The feedback sandwich. J Phys Educ Recreat Dance. 1990;61(9):17-8. http://dx.doi.org/10.1080/07303084. 1990.10604618.

22. Parkes J, Abercrombie S, McCarty T. Feedback sandwiches affect perceptions but not performance. Adv Health Sci Educ Theory Pract. 2013;18(3):397-407. http://dx.doi.org/10.1007/s10459-012-9377-9. PMid:22581568.

23. French JC, Colbert CY, Pien LC, Dannefer EF, Taylor CA. Targeted feedback in the milestones era: utilization of the ask-tellask feedback model to promote reflection and self-assessment. J Surg Educ. 2015;72(6):274-9. http://dx.doi.org/10.1016/j. jsurg.2015.05.016. PMid:26123726.

24. Menachery EP, Knight AM, Kolodner K, Wright SM. Physician characteristics associated with proficiency in feedback skills. J Gen Intern Med. 2006;21(5):440-6. http://dx.doi.org/10.1111/j.1525-1497.2006.00424.x. PMid:16704385.

25. Pricinote SCMN, Pereira ERS. Percepção de discentes de medicina sobre o feedback no ambiente de aprendizagem. Rev Bras Educ Med. 2016;40(3):470-80. http://dx.doi.org/10.1590/1981-52712015v40n3e00422014.

26. Telio S, Regehr G, Ajjawi R. Feedback and the educational alliance: examining credibility judgements and their consequences. Med Educ. 2016;50(9):933-42. http://dx.doi.org/10.1111/medu.13063. PMid:27562893.

27. Lee M, Wimmers PF. Validation of a performance assessment instrument in problem-based learning tutorials using two cohorts of medical students. Adv Health Sci Educ Theory Pract. 2016;21(2):341-57. http://dx.doi.org/10.1007/s10459-0159632-y. PMid:26307371.

28. Baik C, Naylor R, Arkoudis S. The first year experience in Australian universities: findings from two decades 1994-2014. Melbourne: The University of Melbourne; 2015. 
29. Harrison CJ, Konings KD, Schuwirth L, Wass V, van der Vleuten C. Barriers to the uptake and use of feedback in the context of summative assessment. Adv Health Sci Educ Theory Pract. 2015;20(1):229-45. http://dx.doi.org/10.1007/s10459-0149524-6. PMid:24906462.

30. Rudland J, Wilkinson T, Wearn A, et al. A student centred feedback model for educators. Clin Teach. 2013;10(2):99-102. http://dx.doi.org/10.1111/j.1743-498X.2012.00634.x. PMid:23480111.

\section{Contribuição dos autores}

Todos os autores participaram do planejamento e execução do trabalho. 